\title{
Comparison of antimicrobial resistance pattern in hospital versus community-acquired infections in pediatric patients
}

\author{
Mojtaba Hedayat Yaghoobi ${ }^{1(D)}$, Pezhman Karami ${ }^{\text {(D) }}$, Azad Khaledi ${ }^{3,7}$ (D) Elahe Rafie $^{4}$ (D), \\ Mohammadmahdi Sabahi $^{4}$ (D) Behzad Habibipour ${ }^{5}$ (D) Farshid Rahimi-Bashar $^{6}$ (D) Iraj Sedighi $^{7}$
}

1. Dept. of Infectious Disease, Faculty of Medicine, Alborz University of Medical Sciences, Karaj, Iran

2. Dept. of Microbiology, Faculty of Medicine, Hamadan University of Medical Sciences, Hamadan, Iran

3. Dept. of Microbiology, Faculty of Medicine, Kashan University of Medical Sciences, Kashan, Iran

4. Student Researches Committee, School of Medicine, Hamadan University of Medical Sciences, Hamadan, Iran

5. Besat Hospital, Hamadan University of Medical Sciences, Hamadan, Iran

6. Dept. of Anesthesiology and Critical Care, Faculty of Medicine, Hamadan University of Medical Sciences, Hamadan, Iran

7. Dept. of Pediatrics, Faculty of Medicine, Hamadan University of Medical Sciences, Hamadan, Iran

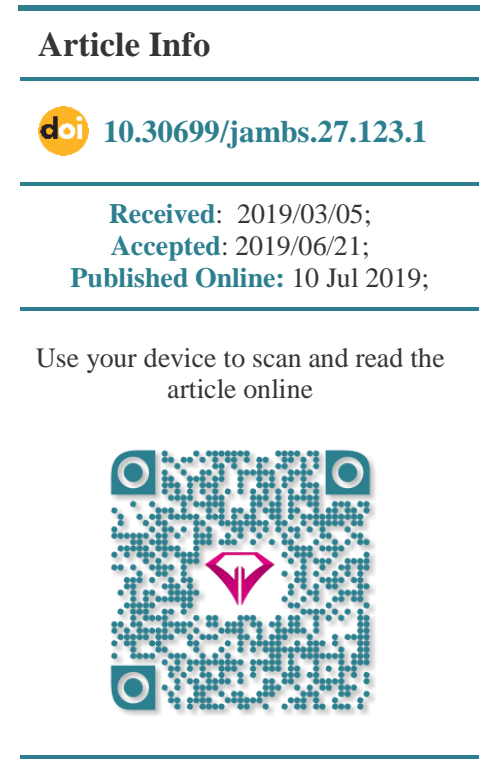

Corresponding Information: Iraj Sedighi, Dept. of Pediatrics, Faculty of Medicine, Hamadan University of Medical Sciences, Hamadan, Iran E-Mail: doctorsedighi@yahoo.com

\section{ABSTRACT}

Background \& Objective: Nosocomial infection is one of the most daunting challenges to the global health care system. The current study aimed to compare the antimicrobial resistance pattern in hospital versus community-acquired infections in pediatric patients in Besat Hospital, Hamadan, Iran.

Materials \& Methods: The current study was carried out on all patients with a hospital-acquired infection (HAI) who were hospitalized in Besat Hospital, Hamadan, Iran, for 48 hours. Moreover, patients with community-acquired infections (who had an infection before hospitalization) were also included in the current study. E-test and Kirby-Bauer disk diffusion test were utilized to determine antimicrobial susceptibility patterns according to the report test/CLSI M100/S27 guidelines. Antibiotic resistance patterns in both patients with hospital and community-acquired infections were separately recorded. Subsequently, the obtained data were analyzed by SPSS software (version 16).

Results: A number of 43 and 68 patients showed HAI and CAI, respectively. The most common HAI and CAI were blood-stream infection and urinary tract infection (UTI) with a prevalence of $69.8 \%$, and $73.5 \%$, respectively. The most frequent organisms isolated from CA and HA-infections were E. coli and Staphylococcus aureus with a prevalence of $67.9 \%$, and $30.2 \%$, respectively. The highest level of resistance in Gram-negative bacteria was observed against cotrimoxazole and ceftriaxone. Furthermore, the highest resistance in Gram-positive organisms was against clindamycin. The prevalence rates of MRSA isolates in HAI and CAI were reported as $46.2 \%$ and $36.9 \%$, respectively.

Conclusion: The obtained results indicated the high resistance to several antibiotics that can be used as promising choices in the treatment of both CA and HA-infections. It is recommended that region-specific monitoring studies be carried out in order to assist the clinician to select the accurate empirical therapy.

Keywords: Pediatric, Drug resistance, Nosocomial infection, Community-acquired infection

\section{Introduction}

Nosocomial infection or a healthcare-associated infection (HAI) is known as one of the serious challenges posed to the global health care system (1). Just 1.7 million HAIs were reported in the USA in 2002(2). It accounts for 99000 deaths and the annual cost of more than 30 million dollars just in the USA (3). Moreover, other countries reported a high frequency of these infections (4). In a nutshell, $8.7 \%$ of the admitted patients around the world are suffering from nosocomial infections (5). In European countries, the prevalence of
HAIs in the general pediatric ward is about $1 \%(1)$, while it was reported as $23.6 \%$ in the neonatal intensive care wards (6). It is worthy to note that other hospital infections are contracted from the application of medical invasive devices or aggressive procedures. Among these infections, the most prevalent HAI is urinary tract infection (UTI) and the most prevalent life-threatening hospital-acquired infection is recognized to be hospitalacquired pneumonia (2). 
In addition to these three important groups of HAIs, infections that are related to the use of invasive devices and surgical wound infections are the common causes of nosocomial infections and mortality resulted from hospitalized patients (7). There are some common causes of concerns in HAI groups of pediatrics and neonates (8). They include defects of skin barriers, immature immune system, particularly in preterm infants, anomalous bacterial defenses and migration of granulocyte, reduction in maternal IgG level in the first months of life, and lower activity of mucous membrane. In the USA, more than $30 \%$ of hospital infections, $70 \%$ of which are contracted in intensive care units (ICU), are caused by Gram-negative bacilli. Enterobacteriaceae are the most common causative microbial agents amongst Gram-negative bacilli which cause hospital-acquired infections (9). Unfortunately, the number of resistant Gram-negative bacilli consisting of Enterobacteriaceae producing extended-spectrum $\beta$-lactamases (ESBLs)or carbapenemases, Pseudomonas aeruginosa, and A. baumannii are on the rise worldwide (1).

It is noteworthy that the prevalence of ESBLproducing Enterobacteriaceae family reached up to $60 \%$ nowadays (10). Furthermore, the rate of nosocomial infections caused by resistant Gram-positive cocci, such as Enterococcus, Staphylococcus has increased to $30 \%$ and $50 \%$, respectively (11). It is of utmost importance to determine the pattern of antimicrobial resistance and its trend in each hospital for the selection of an appropriate empiric antibiotic regimen (12). With this background in mind, the current study aimed at comparing the pattern of antimicrobial resistance between hospital and community-acquired infections during 6 months of 2016 in pediatric cases in Besat Hospital, Hamadan, Iran.

\section{Materials and Methods}

\section{Study population}

This retrospective descriptive cross-sectional survey was carried out in the pediatric intensive care unit (PICU), pediatric hematology, and other pediatric wards of Besat Hospital, Hamadan, during 6 months of 2016.

\section{Inclusion criteria}

During the study period, all patients who had eligible criteria for each kind of nosocomial infection were enrolled in the study using a checklist. The definition of nosocomial infection was similar to that provided by the Centers for Disease Control and Prevention (CDC) (12). To determine the infection type (nosocomial or community-acquired) and clinical samples (tracheal culture, urine, blood, wound, and other samples), all patients (Patients and clinical specimens) were evaluated by the infection control team.

\section{Sampling method}

Census sampling method was used for sampling. Thereafter, cultural process and susceptibility testing were performed on clinical specimens which were sent to the laboratory. All patients hospitalized with an infection and positive culture without the history of recent hospitalization were separately recorded and analyzed to compare the pattern of antibiotic resistance of HAIs with CA infections during this period.

\section{Detection of microorganisms}

After delivering clinical samples to the laboratory, they were immediately cultured on proper media, such as Blood agar, MacConkey agar, EMB, and Nutrient agar and then incubated overnight. After $24 \mathrm{~h}$, the presence of cocci, Bacillus, and Gram-positive/negative bacteria was detected using macroscopic (Colonies shape) and microscopic (Gram-staining) techniques. Subsequently, Catalase/ Oxidase, OF, TSI, Urease, IMViC tests growth on mannitol Salt Agar medium 6\%, Growth/in the presence of salt, growth at 25,37 , and $42^{\circ} \mathrm{C}$ and other microbiology and biochemical tests were used to identify the genus and species of bacteria.

\section{Antibiotic susceptibility testing}

To intent upon antimicrobial susceptibility pattern, the Kirby Bauer Disk Diffusion test was utilized as it was recommended in the CLSI /M100-S27 guidelines (13). Cefoxitin disk diffusion was used to determine methicillin resistance $S$. aurous (MRSA). In addition, E-test was used to determine the susceptibility of bacteria against Meropenem, Colistin, and Vancomycin. The antibiotic disks which were used in the current study were provided from MAST Company (UK). These antibiotic disks included Ceftriaxone $(30 \mu \mathrm{g})$, Ceftazidime $(30 \mu \mathrm{g})$, Cefepime $(30 \mu \mathrm{g})$, Gentamicin $(10 \mu \mathrm{g})$, Ciprofloxacin $(5 \mu \mathrm{g}))$, Co-trimoxazole, Piperaciline-tazobactam, AmpiSulbactame $(20 / 10 \mu \mathrm{g})$, Imipenem $(10 \mu \mathrm{g})$, Meropenem $(10 \mu \mathrm{g})$, Colistin $(10 \mu \mathrm{g})$, Cefazolin $(30 \mu \mathrm{g})$, Erythromycin $(30 \mu \mathrm{g}), \quad$ Clindamycin $(30 \mu \mathrm{g}), \quad$ Cefoxitine $\quad(30 \mu \mathrm{g})$, Cloxacillin $(30 \mu \mathrm{g})$, and Vancomycin.

\section{Statistical analysis}

The obtained data were analyzed in SPSS software (version 16.0) using descriptive statistics. The Ethics Review Board of the Hamadan University of Medical Sciences approved the study. (IR.UMSHA.REC. 1395.352).

\section{Results}

\section{Characterization of study participants}

The present study was conducted on 43 patients who were hospitalized for more than $48 \mathrm{~h}$ in Besat Hospital, Hamadan, Iran, as well as 68 individuals who were hospitalized in the same wards due to community-acquired infections. Notably, $42.6 \%$ and $60.5 \%$ of patients with community-acquired infections and hospital-acquired infections were males, respectively. The mean age of patients with community-acquired infection and hospital infection was reported as 2.17 and 5.27 years, respectively. The most collected samples from patients with communityacquired infections were urine samples with a frequency of $50(73.5 \%)$. On the other hand, most samples achieved 
from patients with hospital-acquired infection were related to blood samples (BSI) with the occurrence of $30(69.8 \%)$.

\section{Main results}

The most common organisms recovered from community-acquired and hospital-acquired infections were E. coli and S. aurous with a prevalence of $67.6 \%$ and $30.2 \%$, respectively (Table 1 ). As evidenced by the findings, the least resistance in the community-acquired infections against $E$. coli was related to Colistin and Meropenem with a prevalence of $0 \%$, followed by Imipenem and Cefepime with the prevalence of $2.2 \%$ and $8.9 \%$, respectively. Conversely, the highest resistance was pertinent to Co-trimoxazole with a frequency of $64.4 \%$, followed by ceftriaxone $(27.6 \%)$. Klebsiella has a high sensitivity to most antibiotics and showed the highest resistance to Co-trimoxazole (75\%). Among hospital-acquired infections caused by E. coli, the minimum resistance was against Colistin, Meropenem, and Gentamicin with a frequency of $0 \%$, followed by piperacillin/tazobactam (10\%). Moreover, the highest resistance in $E$. coli was against Cotrimoxazole with a prevalence of $40 \%$. In addition, in Klebsiella pneumonia isolated from hospital-acquired infections, the minimum resistance was against Colistin, Imipenem, Meropenem, and Piperacillin-tazobactam $(0 \%)$, and $42.9 \%$ of isolates were resistant to Cotrimoxazole.

In Acinetobacter baumannii, the least resistance was to ciprofloxacin with the occurrence of $16.6 \%$, followed by meropenem, gentamycin, colistin, and piperacillintazobactam with the frequency of $33.2 \%$. While, the highest resistance was against Co-trimoxazole (66.7\%), (Table 2). In community-acquired infections caused by S. aurous, the least resistance was to vancomycin $(0 \%)$, followed by ciprofloxacin $(18.2 \%)$, and the highest resistance was to clindamycin with a prevalence of $54.5 \%$. In the case of hospital-acquired infections, Vancomycin was the most effective antibiotic for infections caused by $S$. aurous and the most resistance in this organism was observed against clindamycin with a prevalence of $53.8 \%$. About $36.4 \%$ of $S$. aurous which were retrieved from community-acquired infections was MRSA, as compared to $42.6 \%$ of $S$. aurous isolates which were recovered from hospital-acquired infections. Of these community-acquired MRSA isolates, $45.5 \%$ were resistant against Co-trimoxazole and $66.6 \%$ were resistant to clindamycin.

Notably, no resistance was reported against vancomycin and ciprofloxacin in $S$. aurous isolated from both community and hospital-acquired infections (Table 3). In community-acquired UTI caused by E. coli, the least resistance was to imipenem, meropenem (2.9\%), and piperacillin-tazobactam $(8.9 \%)$, while the highest resistance was related to Cotrimoxazole (74.3\%), followed by ceftriaxone $(28.6 \%)$. In hospital-acquired UTI caused by Escherichia coli (E. coli), the lowest resistance was to Meropenem, piperacillin-tazobactam, and gentamicin $(0 \%)$, and the highest resistance was to Co-trimoxazole $(90 \%)$. In Klebsiella produced community-acquired infection, $100 \%$ of isolates were resistant to Co-trimoxazole.

In general, in Gram-negative bacteria caused by community-acquired-UTI, the highest resistance was to Cotrimoxazole with a prevalence of $71.4 \%$, followed by gentamycin and ceftriaxone with a prevalence of $24.5 \%$, and with no resistance against Colistin and Meropenem. Moreover, the resistance rates against Imipenem and Cefepime were reported as $2 \%$ and $8.2 \%$, respectively. The antibiotic susceptibility results of Gram-negative bacteria which cause bloodstream infection (BSI) indicated that the highest resistance was to Ceftriaxone in community-acquired infections with a prevalence of $31.6 \%$, followed by tazocin and gentamycin with the prevalence of $10.5 \%$. However, no resistance was reported to Colistin. Nonetheless, in Gram-negative bacteria which caused hospital-acquired infections, the most resistance was reported to Ceftriaxone with a frequency of $37.5 \%$ and the least resistance was to Colistin, gentamycin, and tazocin with the prevalence of $12.5 \%$.

In Gram-positive organisms which caused community-bloodstream infections, no resistance was observed to vancomycin $(0 \%)$. Moreover, the resistance rate against ciprofloxacin and cefoxitin was estimated at $16.7 \%$, whereas the highest resistance was to clindamycin (50\%). On the other hand, in Gram-positive organisms which caused hospital-acquired bloodstream infection, the most resistance was to erythromycin with a prevalence of $53.8 \%$, followed by cefoxitin with the prevalence of $46.2 \%$. Nevertheless, no resistance was observed against vancomycin $(0 \%)$. Frequencies of MRSA isolates in community and hospital-acquired bloodstream infections were reported as $16.7 \%$, and $46.2 \%$, respectively.

\section{Discussion}

In The present study demonstrated that the most common organisms isolated from both community and hospitalacquired infections in pediatrics patients were $E$. coli and $S$. aurous with a prevalence of $67.6 \%$ and $30.2 \%$, respectively. These findings were inconsistent with the studies conducted in India (14) and Turkey (15) which found that $E$. coli and $S$. aurous were most prevalent. Moreover, the highest resistance was observed against Co-trimoxazole in $E$. coli which caused both community-acquired and hospitalacquired infections. Therefore, due to its high resistance, Cotrimoxazole is not recommended as the mainstay of treatment for empirical therapy in both types of infections. This could be due to indiscriminate prescribing of these antibiotics to pediatric outpatients. 
Table 1. Oligonucleotide primers used for RT-PCR

\begin{tabular}{|c|c|c|c|c|}
\hline \multicolumn{2}{|r|}{ Variables } & $\begin{array}{l}\text { Community acquired } \\
\text { infection }(\%)\end{array}$ & $\begin{array}{l}\text { Hospital acquired } \\
\text { infection }(\%)\end{array}$ & $\begin{array}{c}\text { p-value } \\
\left(\text { chi }^{2}\right)\end{array}$ \\
\hline \multirow{4}{*}{ Samples } & Urine & $50(73.5)$ & $7(16.3)$ & \multirow{6}{*}{0.001} \\
\hline & Blood & $9(13.2)$ & $30(69.8)$ & \\
\hline & Wound & $5(7.3)$ & $5(11.6)$ & \\
\hline & Other* & $4(5.9)$ & $1(2.3)$ & \\
\hline \multirow{6}{*}{ Microorganisms } & Staphylococcus aurous & $11(16.2)$ & $13(31)$ & \\
\hline & Streptococcus & $2(2.9)$ & $1(2.3)$ & \\
\hline & E. coli & $45(66.2)$ & $10(23.8)$ & \multirow{3}{*}{0.001} \\
\hline & Acinetobacter & $0(0.00)$ & $6(14.3)$ & \\
\hline & Klebsiella & $4(5.9)$ & $7(16.7)$ & \\
\hline & Other & $6(7.3)$ & $5(11.6)$ & \\
\hline
\end{tabular}

*: CSF, secretions, respiratory, Abscess, and Ascites

Table 2. Results of susceptibility test in gram negative organisms based on the infections types

\begin{tabular}{|c|c|c|c|c|c|c|c|c|}
\hline \multirow{2}{*}{ Antibiotics } & \multirow[t]{2}{*}{ Susceptibility } & \multicolumn{3}{|c|}{ Community acquired infection } & \multicolumn{4}{|c|}{ Hospital acquired infection } \\
\hline & & $\begin{array}{l}\text { E.coli } \\
\mathrm{N}=45\end{array}$ & $\begin{array}{c}\text { Klebsiella } \\
\quad \mathrm{N}=4\end{array}$ & $\begin{array}{c}\text { Other } \\
\mathrm{N}=6\end{array}$ & $\begin{array}{c}\text { E. coli } \\
\mathrm{N}=10\end{array}$ & $\begin{array}{c}\text { klebsiella } \\
\mathrm{N}=7\end{array}$ & $\begin{array}{c}\text { Pseudomonas } \\
\text { N=2 }\end{array}$ & $\begin{array}{c}\text { Acinetobacter } \\
\mathbf{N}=6\end{array}$ \\
\hline \multirow{3}{*}{ Ceftriaxone } & $\mathbf{S}$ & $30(68.2)$ & $3(75 / 0)$ & $4(50 / 0)$ & $8(80)$ & $(\% 4.71) 5$ & $2(100.0)$ & $1(16.7)$ \\
\hline & $\mathbf{I}$ & $3(6.7)$ & $1(25)$ & $0(0 / 0)$ & $0(00.0)$ & 0 & $0(00.0)$ & 2(33.3) \\
\hline & $\mathbf{R}$ & $12(27.6)$ & $0(00.0)$ & $2(50 / 0)$ & $2(20)$ & $2(28.6 \%)$ & $0(00.0)$ & $3(50.0)$ \\
\hline \multirow{3}{*}{ Ceftazidime } & $\mathbf{S}$ & $36(80 / 0)$ & $3(75 / 0)$ & $4(50 / 0)$ & $8(80)$ & $6(85.7 \%)$ & $2(100.0)$ & $3(50.0)$ \\
\hline & $\mathbf{I}$ & $4(8.9)$ & $1(25 / 0)$ & $1(25 / 0)$ & $0(00.0)$ & 0 & $0(00.0)$ & $0(00.0)$ \\
\hline & $\mathbf{R}$ & $5(11 / 1)$ & $0(00.0)$ & $1(25 / 0)$ & $2(20 / 0)$ & $1(14.3)$ & $0(00.0)$ & $3(50.0)$ \\
\hline \multirow{3}{*}{ Cefepime } & $\mathbf{S}$ & $37(82 / 2)$ & $4(100.0)$ & $4(50 / 0)$ & $8(80 / 0)$ & $6(85.7 \%)$ & $2(100.0)$ & $3(50.0)$ \\
\hline & $\mathbf{I}$ & $4(8.9 \%)$ & $0(00.0)$ & $1(25 / 0)$ & $0(00.0)$ & 0 & $0(00.0)$ & $1(16.7)$ \\
\hline & $\mathbf{R}$ & $4(8.9 \%)$ & $0(00.0)$ & $1(25 / 0)$ & $2(20 / 0)$ & $1(14.3)$ & $0(00.0)$ & $2(33.3)$ \\
\hline \multirow{3}{*}{ Gentamicin } & $\mathbf{S}$ & $33(73.3)$ & $4(100.0)$ & $3(50.0)$ & $10(100.0)$ & $5(71.4 \%)$ & $2(100.0)$ & $4(66.7)$ \\
\hline & $\mathbf{I}$ & $1(2.2 \%)$ & 0 & 0 & 0 & $1(14.3)$ & 0 & 0 \\
\hline & $\mathbf{R}$ & $11(24.4)$ & $0(00.0)$ & $3(50 \%)$ & $0(00.0)$ & $1(14.3)$ & $0(00.0)$ & $2(33.3)$ \\
\hline \multirow{3}{*}{ Ciprofloxacin } & $\mathbf{S}$ & $37(82.2)$ & $3(75 / 0)$ & $5(75 / 0)$ & $7(70 / 0)$ & $6(85.7 \%)$ & $2(100 / 0)$ & $4(66.7)$ \\
\hline & $\mathbf{I}$ & $0(00.0)$ & $0(0.0)$ & $0(00.0)$ & $0(0.0)$ & 0 & $0(00.0)$ & $1(16.7)$ \\
\hline & $\mathbf{R}$ & $8(17 / 8)$ & $1(25 / 0)$ & $1(25 / 0)$ & $3(30 / 0)$ & $1(14.3)$ & $0(00.0)$ & $1(16.6)$ \\
\hline \multirow{3}{*}{ Co-trimoxazole } & $\mathbf{S}$ & $16(35.6)$ & $1(25 / 0)$ & $4(75 / 0)$ & $6(60 / 0)$ & $4(57.1)$ & $1(50 / 0)$ & $1(16.6)$ \\
\hline & $\mathbf{I}$ & $0(0 / 0)$ & $0(0 / 0)$ & $0(0 / 0)$ & $0(00.0)$ & 0 & $0(00.0)$ & $1(16.6)$ \\
\hline & $\mathbf{R}$ & $29(64.4)$ & $3(75 / 0)$ & $2(25 / 0)$ & $4(40 / 0)$ & $3(42.9)$ & $1(50 / 0)$ & $4(66.7)$ \\
\hline \multirow{2}{*}{ Piperaciline-tazobactame } & $\mathbf{S}$ & $39(86 / 7)$ & $4(100.0)$ & $6(100.0)$ & $9(90 / 0)$ & $7(100)$ & $(100.0) 2$ & $4(66.7)$ \\
\hline & $\mathbf{R}$ & $6(13 / 3)$ & $0(00.0)$ & $0(00.0)$ & $1(10 / 0)$ & 0 & $0(00.0)$ & $2(33.3 \%)$ \\
\hline \multirow{3}{*}{ Ampi-Sulbactame } & $\mathbf{S}$ & $7(63.6)$ & $1(50.0)$ & $1(50.0)$ & $3(37.5)$ & $5(71.4 \%)$ & $1(50 / 0)$ & $2(33.3)$ \\
\hline & $\mathbf{I}$ & $3(27.3)$ & $1(50.0)$ & $1(50 / 0)$ & $4(50.0)$ & 0 & $1(50 / 0)$ & $0(00.0)$ \\
\hline & $\mathbf{R}$ & $1(9.1)$ & $0(00.0)$ & $0(00.0)$ & $1(12.5)$ & $2(28.6 \%)$ & $0(00.0)$ & $4(66.7)$ \\
\hline \multirow[b]{2}{*}{ Imipenem } & $\mathbf{S}$ & $44(97 / 8)$ & $3(75 / 0)$ & $5(75 / 0)$ & $8(80 / 0)$ & $7(100)$ & $2(100 / 0)$ & $3(50.0)$ \\
\hline & $\mathbf{R}$ & $1(2.2)$ & $1(25.0)$ & $0(0 / 0)$ & $2(20.0)$ & 0 & $0(00.0)$ & $3(50.0)$ \\
\hline \multirow{3}{*}{ Meropenem } & $\mathbf{S}$ & $45(100.0)$ & $4(100.0)$ & $6(100.0)$ & $9(90.0)$ & $7(100)$ & $2(100.0)$ & $3(50.0)$ \\
\hline & I & $0(0 / 0)$ & $0(0.0)$ & $0(0 / 0)$ & $0(7 / 69)$ & 0 & $0(00.0)$ & $1(7 / 16)$ \\
\hline & $\mathbf{R}$ & $0(0 / 0)$ & $0(0 / 0)$ & $0(0 / 0)$ & $1(10.0)$ & 0 & $0(00.0)$ & $2(2 / 33)$ \\
\hline \multirow{2}{*}{ Colistin } & $\mathbf{S}$ & $(100.0) 45$ & $4(100.0)$ & $6(100.0)$ & $(100.0) 10$ & $7(100)$ & $2(100.0)$ & $4(66.7 \%)$ \\
\hline & $\mathbf{R}$ & $0(0 / 0)$ & $0(00.0)$ & $0(00.0)$ & $0(0 / 0)$ & 0 & $0(00.0)$ & $2(33.3 \%)$ \\
\hline
\end{tabular}


Table 3. Results of susceptibility testing in gram positive organisms based on the infections

\begin{tabular}{|c|c|c|c|c|c|c|c|}
\hline \multirow{2}{*}{ Antibiotics } & \multirow{2}{*}{ Susceptibility } & \multicolumn{3}{|c|}{ Community acquired infection } & \multicolumn{3}{|c|}{ Hospital acquired infection } \\
\hline & & $\begin{array}{c}\text { Other } \\
\mathrm{N}=2\end{array}$ & $\begin{array}{l}\text { S. aurous } \\
\mathrm{N}=11\end{array}$ & $\begin{array}{c}\text { Streptococcus } \\
\mathrm{N}=2\end{array}$ & $\begin{array}{c}\text { Other } \\
\mathrm{N}=1\end{array}$ & $\begin{array}{l}\text { S. aurous } \\
\mathrm{N}=13\end{array}$ & $\begin{array}{c}\text { Streptococcus } \\
\mathrm{N}=1\end{array}$ \\
\hline \multirow{2}{*}{ Cefazolin } & $\mathbf{S}$ & $1(50.0)$ & $8(72.7)$ & $2(100.0)$ & $1(100.0)$ & $9(69.2)$ & $0(00.0)$ \\
\hline & $\mathbf{R}$ & $2(50.0)$ & $3(23.7)$ & $0(00.0)$ & $0(00.0)$ & $4(30.8)$ & $1(100.0)$ \\
\hline \multirow{3}{*}{ Erythromycin } & $\mathbf{S}$ & $1(50.0)$ & $5(45.5)$ & $1(50.0)$ & $1(100.0)$ & $4(30.8)$ & $0(00.0)$ \\
\hline & $\mathbf{I}$ & $0(00.0)$ & $0(00.0)$ & $0(00.0)$ & $0(00.0)$ & $1(7.7)$ & $0(00.0)$ \\
\hline & $\mathbf{R}$ & $1(50.0)$ & $6(54.5)$ & $1(50.0)$ & $0(00.0)$ & $8(61.5)$ & $1(100.0)$ \\
\hline \multirow{3}{*}{ Clindamycin } & $\mathbf{S}$ & $1(50.0)$ & $3(27.3)$ & $1(50.0)$ & $1(100.0)$ & $6(46.2)$ & $1(50.0)$ \\
\hline & I & $0(00.0)$ & $1(9.1)$ & $0(00.0)$ & $0(00.0)$ & $0(00.0)$ & $0(00.0)$ \\
\hline & $\mathbf{R}$ & $1(50.0)$ & $7(63.6)$ & $1(50.0)$ & $0(00.0)$ & $7(53.83)$ & $0(00.0)$ \\
\hline \multirow{3}{*}{ Ciprofloxacin } & $\mathbf{S}$ & $1(50.0)$ & $7(63.6)$ & $1(50.0)$ & $1(100.0)$ & $10(76.9)$ & $0(00.0)$ \\
\hline & I & $0(00.0)$ & $2(18.2)$ & $1(50.0)$ & $0(00.0)$ & $0(00.0)$ & $1(100.0)$ \\
\hline & $\mathbf{R}$ & $1(100.0)$ & $2(18.2)$ & $0(00.0)$ & $0(00.0)$ & $3(23.1)$ & $0(00.0)$ \\
\hline \multirow{2}{*}{ Cotrimoxazole } & $\mathbf{S}$ & $1(50.0)$ & $6(54.5)$ & $1(50.0)$ & $0(00.0)$ & $8(61.5)$ & $0(00.0)$ \\
\hline & $\mathbf{R}$ & $1(50.0)$ & $5(45.5)$ & $2(100.0)$ & $1(100.0)$ & $6(46.2)$ & $0(00.0)$ \\
\hline \multirow{3}{*}{ Cefoxitine } & $\mathbf{S}$ & $2(100.0)$ & $6(54.5)$ & $3(75.0)$ & $1(100.0)$ & $2(66.7)$ & $3(75.0)$ \\
\hline & I & $0(00.0)$ & $1(9.1)$ & $0(00.0)$ & $0(00.0)$ & $1(7.7)$ & $0(00.0)$ \\
\hline & $\mathbf{R}$ & $0(00.0)$ & $4(36.4)$ & $0(00.0)$ & $0(00.0)$ & $6(46.2)$ & $1(100.0)$ \\
\hline \multirow{3}{*}{ Cloxacillin } & $\mathbf{S}$ & $1(50.0)$ & $4(36.4)$ & $1(50.0)$ & $1(100.0)$ & $8(61.5)$ & $0(00.0)$ \\
\hline & $\mathbf{I}$ & $0(00.0)$ & $2(18.2)$ & $0(00.0)$ & $0(00.0)$ & $0(00.0)$ & $0(00.0)$ \\
\hline & $\mathbf{R}$ & $1(50.0)$ & $5(45.5)$ & $1(50.0)$ & $0(00.0)$ & $5(38.5)$ & $1(100.0)$ \\
\hline Vancomycin & $\mathbf{S}$ & $2(100.0)$ & $11(100.0)$ & $2(100.0)$ & $1(100.0)$ & $13(100.0)$ & $1(100.0)$ \\
\hline
\end{tabular}

\section{Discussion}

In The present study demonstrated that the most common organisms isolated from both community and hospital-acquired infections in pediatrics patients were E. coli and $S$. aurous with a prevalence of $67.6 \%$ and $30.2 \%$, respectively. These findings were inconsistent with the studies conducted in India (14) and Turkey (15) which found that $E$. coli and $S$. aurous were most prevalent. Moreover, the highest resistance was observed against Co-trimoxazole in $E$. coli which caused both community-acquired and hospital-acquired infections. Therefore, due to its high resistance, Cotrimoxazole is not recommended as the mainstay of treatment for empirical therapy in both types of infections. This could be due to indiscriminate prescribing of these antibiotics to pediatric outpatients.

As evidenced by the obtained results, no resistance was found against Colistin and Meropenem in Gramnegative microorganisms which caused the communityacquired infections, followed by Imipenem and
Cefepime with the prevalence of $2.2 \%$, and $8.9 \%$, respectively. Consequently, Meropenem can be used as the first-line treatment empirically in critically ill patients with HA-sepsis until obtaining the results of culture and susceptibility test. However, in CAinfections, cefepime can be used as an acceptable antibiotic in the empirical regime. In A. baumannii, the minimum of resistance was reported against ciprofloxacin which was probably owing to the no or at least use of this antibiotic in the pediatric ward. In CA and HA-infections caused by Gram-positive cocci, no resistance was observed against vancomycin; however, $36.4 \%$ of CA-S. aurous and $42.6 \%$ of HA-S. aurous were resistant to cefoxitin (MRSA).In addition, the highest resistance in $S$. aurous was reported against clindamycin which indicates that these medicines should not be prescribed in empirical therapy.

There exists a high resistance of methicillin in MRSA strains with a high percentage of prevalence in hospitalized 
patients suspected to Gram-positive infectious agents. In this case, vancomycin empirical prescription and utilization should be undertaken until getting the results of culture and susceptibility testing. In community-acquired infections which are caused by E. coli, the highest resistance was to Cotrimoxazole with a frequency of $64.4 \%$, followed by Ceftriaxone (27.6\%). In line with these results, a study conducted in Tehran in 2007 found that the most common organism was $E$. coli with a prevalence of $51.4 \%$, and the most resistance was against Ampicillin and the least one was to ciprofloxacin, ofloxacin, and amikacin (16). Whereas, in a study performed in Bahrami Hospital, Tehran, the most common reported isolates were P. aeruginosa $(24.3 \%)$ and Klebsiella pneumonia (18.6\%) (17). The prevalence of pathogenesis is roughly similar in the distinct regions all around the world despite the fact that antimicrobial resistance patterns are markedly different.

This difference in antibiotic resistance patterns in various parts of the world is attributed to the immoderate and infelicitous antibiotics utilization, geographical difference, and the difference in prescribing the antibiotic regimes (15). E. coli was recognized as the most prevalent organism in UTI which is consistent with the results of previous studies $(18,19)$. Moreover, our findings revealed that $E$. coli isolated from UTI in both CA and HA infections had the same antimicrobial pattern since Imipenem was the most active agent against $E$. coli and the most resistant one to Cotrimoxazole. In the present study, $100 \%$ of $K$. pneumonia isolated from community-acquired infections were resistant to Co-trimoxazole. Whereas, all isolates were susceptible to almost all antibiotics in acquired-hospital infections. In accordance with these results, a study conducted by Yuksel et al. (2006) conducted on UTI in Turkey revealed that the most common causative agent was $E$. coli, followed by $K$. pneumonia. Moreover, resistance to ampicillin and $\mathrm{Co}$ trimoxazole in all isolates was another noteworthy result of the mentioned study (20). In community-acquired UTI, we reported $100 \%$ resistance against Co-trimoxazole; nonetheless, it should be omitted from the drug regime in the treatment of children-UTI. Instead, the low level of resistance to Imipenem and Meropenem can be a reasonable alternative in the treatment of UTI $(20,21)$.

In community-acquired bloodstream infection (CA-BSI) caused by Gram-negative bacteria, the highest resistance was reported against ceftriaxone with a prevalence of $31.6 \%$, while no resistance was described against Colistin. Whereas in hospital-acquired bloodstream infection (HA-BSI), the most resistance was against ceftriaxone with a prevalence of $37.5 \%$ and the lowest was against Colistin/gentamicin and tazocin with a frequency of $12.5 \%$. Consequently, the prescription of gentamicin or Piperacillin/Tazobactam rather than ceftriaxone can be the proper choice in BSI cases.
Furthermore, in Gram-positive organisms which caused CA$\mathrm{BSI}$, the least resistance was against vancomycin $(0 \%)$, while the highest was to clindamycin (50\%). Nevertheless, among Gram-positive organisms which developed HA-BSI, the most resistance was to cefoxitin with a prevalence of $46.2 \%$ and the least resistance to vancomycin $(0 \%)$. Therefore, in patients with HA-BSI, vancomycin can be a suitable choice for empirical treatment. In the current study, MRSA which caused community and hospital-acquired bloodstream infections was reported as $16.7 \%$ and $46.2 \%$, respectively. However, the reported prevalence of MRSA was $79 \%$ in a study (five-year evaluation) conducted by Pourakbari et al. in Iran within 2001-2005. This prevalence was very higher than the value obtained in the current study; moreover, the mentioned study was performed on the patients who suffered from BSI (22).

As mentioned earlier, the antimicrobial pattern of BSI caused by gram-negative isolates in community-acquired infections was consistent with the hospital-acquired one. The best choice for antibiotic therapy was Imipenem or Meropenem, whereas ceftriaxone seemed to be ineffective. In Gram-positive cocci, the most resistance was observed to clindamycin and erythromycin and the most susceptibility was detected to vancomycin. In a study carried out in Nepal within 2006-2008 on neonatal sepsis, the most frequent organisms isolated from blood culture samples were Klebsiella, followed by S. aurous. Moreover, the most effective antibiotic against Gram-negative organisms was cefoperazone/sulbactam and vancomycin in Gram-positive bacteria (23). As illustrated by the obtained results, a number of factors have increased the resistance against antimicrobial drugs. They include adequate staff, state health laws, hygiene in medical processes, control measures, stringent hospital policy regarding diagnosis and treatment of hospital-acquired infections, ongoing training and education, limited medical methods and medical device use, proper application of disinfectants-washers, and antimicrobial medicines (24). Geographic variations in the prevalence of pathogens and profiles of antibiotic susceptibility necessitate continuous monitoring and lead to the selection of best therapeutic options (25).

Taking everything into account, the present study pointed to the high resistance of Gram-negative isolates against Cotrimoxazole and ceftriaxone, as well as clindamycin in Gram-positive isolates. Consequently, it can be used as a good guide for describing the empirical treatment in CA and HA infections. Moreover, the results suggested that Cefepime can be a promising choice for empirical treatment in UTI cases. Furthermore, a combination of gentamycin and vancomycin can be used as an empirical regime in hospitalsepsis.

Journal of Advances in Medical and Biomedical Research 


\section{Conclusion}

In addition, the findings of the current study were indicative of the high resistance to several antibiotics that can be used as excellent choices in the treatment of both CA and HA-infections. On a final note, it is recommended that region-specific monitoring studies be carried out in order to assist the clinician to select the accurate empirical therapy.

\section{Acknowledgements}

The authors thank all those who helped them writing this paper.

\section{Conflict of Interest}

Authors declared no conflict of interests.

\section{References}

1. Falahi J, Khaledi A, Esmaeili D, Ghazvini K, Rostami H. Prevalence and types of bacteria associated with neonatal sepsis in neonatal ward from Ghaem hospital of Mashhad, Iran. Der Pharm Lett.2016; 8 (3) : 240-45.

2. Peleg AY, Hooper DC. Hospital-acquired infections due to gram-negative bacteria. N Engl J Med.2010; 362 (19) : 1804 13. [DOI:10.1056/NEJMra0904124]

3. Anderson DJ, Pyatt DG, Weber DJ, Rutala WA. Statewide costs of health care-associated infections: estimates for acute care hospitals in North Carolina. Am J Infect Control.2013; 41(9):764-8. [DOI:10.1016/i.ajic.2012.11.022]

4. Rezai MS, Bagheri-Nesami M, Nikkhah A, Bayg A. Incidence, risk factors, and outcome of ventilator-associated Pneumonia in 18 hospitals of Iran. Running title: Ventilatorassociated pneumonia in Iran. J Adv Biotechnol Res.2016; 7(3):936-46.

5. Behzadnia S, Davoudi A, Rezai MS, Ahangarkani F. Nosocomial infections in pediatric population and antibiotic resistance of the causative organisms in north of iran. Iran Red Crescent Med J.2014; 16(2): e14562 [DOI:10.5812/ircmj.14562]

6. Raymond J, Aujard Y, Group ES. Nosocomial infections in pediatric patients a European, multicenter prospective study. Infect Control Hosp Epidemiol.2000; 21(4):260-3 [DOI:10.1086/501755]

7. Kirby JP, Mazuski JE. Prevention of surgical site infection. Surg Clin North Am.2009; 89(2):365-89. [DOI:10.1016/j.suc.2009.01.001]

8. Drakulovic MB, Torres A, Bauer TT, Nicolas JM, Nogué S, Ferrer M. Supine body position as a risk factor for nosocomial pneumonia in mechanically ventilated patients: a randomised trial. The Lancet.1999; 354(9193):1851-8 [DOI:10.1016/S0140-6736(98)12251-1]

9. Karakoc C, Tekin R, Yeşilbağ Z, Cagatay A. Risk factors for mortality in patients with nosocomial gram-negative rod bacteremia. Eur Rev Med Pharmacol Sci.2013; 17(7):951-7.

10. Falagas ME, Kastoris AC, Kapaskelis AM, Karageorgopoulos DE. Fosfomycin for the treatment of multidrug-resistant, including extended-spectrum $\beta$ lactamase producing, Enterobacteriaceae infections: a systematic review. Lancet Infect Dis.2010; 10(1):43-50. [DOI:10.1016/S1473-3099(09)70325-1]

11. Minhas P, Perl TM, Carroll KC, et al. Risk factors for positive admission surveillance cultures for methicillinresistan t Staphylococcus aureus and vancomycin-resistant enterococci in a neurocritical care unit. Crit Care Med.2011; 39(10):2322-9. [DOI:10.1097/CCM.0b013e3182227222]

12. Dellit TH, Owens RC, McGowan JE, et al. Infectious Diseases Society of America and the Society for Healthcare Epidemiology of America guidelines for developing an institutional program to enhance antimicrobial stewardship. Clin Infect Dis.2007; 44(2):159-77. [DOI:10.1086/510393]

13. Wikler MA. Performance standards for antimicrobial susceptibility testing Sixteenth informational supplement. 16ate M 100-S 16.2006.

14. Roy MP, Gaind R, Aggarwal KC, Chellani HK, Biswal I. Pattern of pediatric bacterial infection and antibiotic resistance in New Delhi. Indian Pediatr.2017; 54:153-4. [DOI:10.1007/s13312-017-1022-5]

15. Yilmaz Y, Tazegun ZT, Aydin E, Dulger M. Bacterial uropathogens causing urinary tract infection and their resistance patterns among children in Turkey. Iran Red Crescent Med J.2016; 18:4-7 [DOI:10.5812/ircmj.26610]

16. Rastegar Lari A, Karimi A, Razavi Sh, Mostafavi $\mathrm{H}$, Farzandy Gh. Antibiotic resistance of Gram-negative rods isolated from patients in pediatric hospital in Tehran. Iranian J Med Microb. 2007;1(1):67-9.

17. Barak M, Mamishi S, Siadati S A, Salamati P, Khotaii G, Mirzarahim M. Risk factors and bacterial etiologies of nosocomial infections in NICU and PICU wards of children's medical center and Bahrami hospitals during 2008-2009. Ardabil J Univ Med Sci. 2011; 11:113-20.

18. Andrews JM. The development of the BSAC standardized method of disc diffusion testing. J Antimicrob Chemother.2001; [DOI:10.1093/jac/48.suppl_1.29]

19. Mangiarotti P, Pizzini C, Fanos V. Antibiotic prophylaxis in children with relapsing urinary tract infections. J Chemother.2000; 12(2):115-23. [DOI:10.1179/joc.2000.12.2.115]

20. Yüksel S, Öztürk B, Kavaz A, et al. Antibiotic resistance of urinary tract pathogens and evaluation of empirical treatment in Turkish children with urinary tract infections. Int $\mathrm{J}$ Antimicrob Agents. $2006 \quad$;28(5):413-6. [DOI:10.1016/j.ijantimicag.2006.08.009]

21. Haller M, Brandis M, Berner R. Antibiotic resistance of urinary tract pathogens and rationale for empirical intravenous therapy. Pediatr Nephrol.2004; 19(9):982-6. [DOI:10.1007/s00467-004-1528-7]

22. Pourakbari B, Sadr A, Ashtiani MTH, et al. Five-year evaluation of the antimicrobial susceptibility patterns of bacteria causing bloodstream infections in Iran. J Infect Develop Count.2012; 6:120-5. [DOI:10.3855/iidc.1517]

23. Takpere A, Kamble V. Bacterial isolates, risk factors and antibiogram of neonatal septiceamia. Int $\mathrm{J}$ Pharm Bio Sci.2014; 5(1):788-93.

24. Yunita M, Widowati $\mathrm{T}$, Rusmawatiningtyas D. Initial caloric administration as a risk factor for mortality in critically-ill children. Critic Care Shock.2018; 21(2):53-62 
25. Dias Neto JA, Martins ACP, Silva LDMd, et al. Community acquired urinary tract infection: etiology and bacterial susceptibility. Acta Cirurgica Brasileira.2003; 18:33-6. [DOI:10.1590/S0102-86502003001200012]

\section{How to Cite This Article:}

Hedayat Yaghoobi M, Karami P, Khaledi A, Rafie E, Sabahi M, Habibipour B, et al . Comparison of antimicrobial resistance pattern in hospital versus community-acquired infections in pediatric patients. J Adv Med Biomed Res. 2019; 27 (123):1-8

\section{Download citation:}

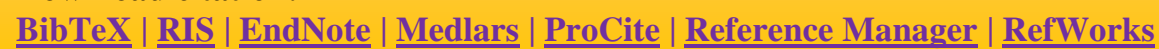

\section{Send citation to:}

9. Mendeley 2 Zotero ;)RefWorks RefWorks 